\title{
Blood droplet dynamics and its forensic implication in crime scene investigation
}

\begin{abstract}
A technique of calculating the origin of blood and trails formed at the crime scene that could give impact directions and positions had been presented in this paper. The size of bloodstains, the volume of droppers, and spikes around the stain border relayed on droplet impact velocity and droplet diameter. It was not unusual to find bloodstain patterns in a violent encounter, and through proper interpretation they could provide very critical facts about such an incident. Precise measurements and digital camerawork might estimate the free height of passive droplets, the features related to the effect of the surface, and the intensity involved in bloodshed. Four diverged forms of stains were made and human blood with heparin as an anti-coagulant was taken. The blood volumes taken were: 10 i 1, 5 ïl, $1 \mathrm{i} € 1,0.5 \mathrm{i}, 0.25 \mathrm{i}$, and $0.1 \mathrm{ï} l$. Pipette (Eppendorf) and precision syringe were applied for computing and discharging the blood splashing. Blood droplets were permitted to fall freely by hand by pressing the needle of the syringe very gently so that drops were detached from the tip of a stainless steel hypodermic needle at their own form. Individually, all volumes were cycled for successively for five times to make four kinds of contact stains and spatter stains on different surfaces used. The results were deduced through Regression Coefficient Relation, One-Way ANOVA and Two-Way ANOVA which showed significance statistically. The resulting stains were than examined and photographed with digital camera.
\end{abstract}

Volume 6 Issue 2 - 2018

\author{
Shahid Iqbal, Akhtar Ali,' Muhammad Ashraf \\ Tahir, ${ }^{2}$ Muhammad Tayyab,' Muhammad \\ Imran,' Khalid Sajjad Feras ${ }^{2}$ \\ 'Institute of Biochemistry and Biotechnology, University of \\ Veterinary \& Animal Sciences, Pakistan \\ 2Punjab Forensic Science Agency, Pakistan
}

Correspondence: Shahid Iqbal, Institute of Biochemistry and Biotechnology, University of Veterinary and Animal Sciences, Lahore, Pakistan, Tel 00923346790679 ,

EmailshahidiqbalII43@yahoo.com

Received: February 19, 2018| Published: March 09, 2018

Keywords: bloodstain pattern analysis, forensic science, impact pattern, bloodletting incidents, hypodermic needle

\section{Introduction}

Bloodstain pattern analyses (BPA) were the most significant proofs found at a crime scene and several indications could be drawn from them. Forensic scientist's evaluated bloodstains by visualizing DNA or defining the blood group with electrophoretic and immunological methods (like identification, species, etc.). Nevertheless, BPA had a relevant significance that sometimes is more vital than the techniques. In this study, bloodstain patterns provide key facts to measure the crimes and actions taken place as a result. BPA had taken the forensic scientists to re-constitute the actions and analyzed the reports of crime followers and witnesses. ${ }^{1}$ BPA was a unit of forensic manners derived on viewing bloodstain initiated in a crime scene with a vision to reform the actions important to these bloodstains. The notion was to find out the reason of the bloodstain, frequently discussed as the bloodletting incident. The main purpose of BPA was to find out a relation of the single blood droplet from the emergent. In the estimation that blood drops emerged from a bloodletting incident were accountable for a particular blood stain, the trails of theses blood drops could join when found back from the bloodstain and the region of meeting of these tracks is thought to give a upright sign of the place of the bloodletting occasion. ${ }^{2}$ Bloodstain pattern study comprises the scientific study of fixed significances causing from forceful blood detaching occasions. A broad learning of bloodstain patterns at crime scene rottenly grows vital proofs. The scattering, size and character of bloodstain on an object, on a suspicious, on the walls, floor, ceilings or on objects on the sight can support reforming these blood shedding measures. Bloodstain pattern study also support one determining the truthfulness of reports given by the observer, target or a suspect. ${ }^{3}$

BPA was the analysis of methods and the alignment and sprinkling of bloodstain projects in accordance to give a explaining of the physical activities of a crime which gave development to their occurrence. The bloodstain patterns could give vital proof of the actions which led to their making when examined by a qualified professional. The material grown could then be used for the improvement of an event and the evaluation of reports of observers and crime providers. BPA was a vital forensic procedure in addition to autopsies, basic crime scene effort and molecular natural science. The genetic analysis mostly was a dynamic part and a highest prerequisite for a compact analysis of bloodstain patterns. The use of these scientific measures, physical sign, usual thoughts, and their interrelationships were the main confidences to achieve obvious knowledge of the series of happenings that border the instruction of a crime. ${ }^{4}$ Several of the incidents of malicious crimes contained bloodstains. Blood spatter stains resulted when drops passively owing to pressure being function to the body. There was a well-known however very miserable process by which a mainly skilled forensic technician could analyze the single blood spots. This mode assessed the body's two dimensional location on the floor plan once the body was clasped. The spitting image study process gave a planned and open another that could be suppressed at crime scenes, assuming the stains were known to be the outcome of spatter. $^{5}$

\section{Materials and methods}

The simple new method for creating bloodstains under identified impact circumstances is properly direct though care prerequisite to be taken at several points to ensure the reproducibility of results. To examine the blood spatter pattern, a sack $(500 \mathrm{~mL})$ blood specimen with heparin was taken from Blood Bank, Ittefaq Hospital, and Lahore. Two beakers $(50 \mathrm{~mL}$ and $100 \mathrm{~mL}$ ) were used as container and to distribute the blood sample. Eppendroff pipette and syringes were used for computing and discharging the bloody stain so the characters 
and patterns could be seen. Human blood with heparin as anticoagulant was availed to give the real and genuine touch to the scène. Substrates chosen for the blood spattering analyses were white paper, cotton cloth, fabricated carpet, wooden slabs metallic road and different tools. At the end digital camera and ABFO (American Board of Forensic Odontology) were used for the photography and measuring scale was also used for the measurement of blood drops respectively.

\section{Experimental design}

Bloodstains frequently take place on roads, clothing, carpets, timber objects, walls and papers. So, this study was conducted on these substrates for four different forms of stains; direct contact stains, indirect contact stains, pressure contact stains and smear contact stains. For staining human blood with heparin as an anticoagulant was used. ${ }^{6}$ The blood volumes of different ranges were used. These volume ranges were measured and released using a pipette (Eppendorf) and also by precision syringe. A syringe fastened to a laboratory stand so that its height above a test surface resting on a flat lab bench would be different. Blood drops were allowed to fall freely by manual depressing of the pippete and the needle of the syringe very gently so that drops separated from the tip of a stainless steel, hypodermic needle under their own mass. Two other different droppers i.e. eye dropper and medicine dropper were used to produce a range of drop sizes magnitudes and the averaged diameter of drops. Each volume was used ten times to produce ten forms of contact stains and spatter stains on various surfaces used in the study. Several series of falling horizontal $\left(90^{\circ}\right.$ impact angle) and projected $\left(10^{\circ}-60^{\circ}\right.$ impact angle) droplets of changed volumes and different heights were produced. The stains made were tested and photographed through digital camera.

\section{Results}

All analyzed bloodstains were located at eleven possible centres of origins. It was noted that as the surface region of the point of the tool from where the blood drops falls rises, the diameter of the blood stain also accelerates and vice versa. Figure 1 indicates that bloodstains fallen from different origins after an interval of every five minutes at a constant height of $100 \mathrm{~cm}$. The height was kept the same, i.e., 100 $\mathrm{cm}$ above the ground. Furthermore, it was also noted that as the diameter of the stain increased, it resulted in the increased volume of the blood drop provided that the height in each case was same. So, it was concluded that the volume of the blood drops depends upon the surface area of the source object, greater the surface area, i.e., origin, larger the volume of the blood drop.

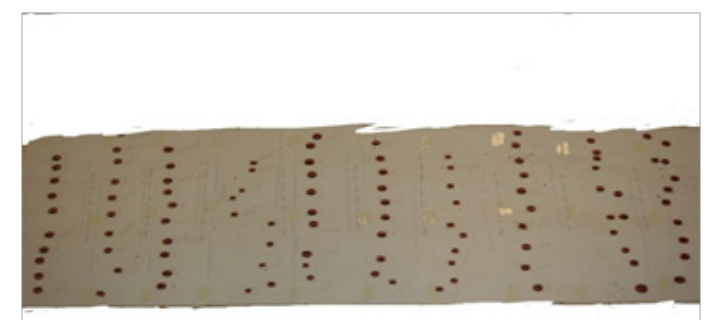

Figure I Blood stains fallen from different origins at a constant height of $100 \mathrm{~cm}$.

\section{Diameter of stain vs. distance fallen and diameter of stain vs. distance of drop}

As the height was increased the diameter of the individual stain got increased also but at the height of $200 \mathrm{~cm}$ and above no further increased in the diameter took place rather minimum volume of the difference of the blood drops gave almost the same diameter stain at $200 \mathrm{~cm}$ and above. The effect of minimum volume difference was liable to be noted below $200 \mathrm{~cm}$ height. The Table 1 shows that when blood fallen at height of $10 \mathrm{~cm}$ with eye dropper with diameter of $0.032 \mathrm{ml}$ then it covered $10.5 \mathrm{~mm}$ surface, pipette dropper with diameter of $0.045 \mathrm{ml}$ then it covered $11.6 \mathrm{~mm}$ surface and medicine dropper with diameter of $0.053 \mathrm{ml}$ then it covered $11.60 \mathrm{~mm}$ surface and similarly others (Figure $2 \&$ Figure 3).

Table I Distance fallen in $\mathrm{cm}$ versus volume of different dropper in $\mathrm{mm}$

\begin{tabular}{llll}
\hline $\begin{array}{l}\text { Distance } \\
\text { fallen in } \mathbf{~ c m}\end{array}$ & $\begin{array}{l}\text { Eye dropper } \\
\mathbf{0 . 0 3 2} \mathbf{m l} \\
\text { diameter in } \\
\mathbf{m m}\end{array}$ & $\begin{array}{l}\text { Pipette } \\
\text { dropper } \\
\mathbf{0 . 0 4 5 m l} \\
\text { diameter in } \\
\mathbf{m m}\end{array}$ & $\begin{array}{l}\text { Medicine } \\
\text { dropper } \\
\mathbf{0 . 0 5 3} \mathbf{m l} \\
\text { diameter in } \\
\mathbf{m m}\end{array}$ \\
\hline 10 & 10.5 & 11.6 & 11.6 \\
25 & 12.7 & 12.6 & 13.1 \\
50 & 14.2 & 14.4 & 15.1 \\
100 & 16.2 & 17 & 17 \\
200 & 18.8 & 18.9 & 19 \\
300 & 17.99 & 19 & 19 \\
\hline
\end{tabular}

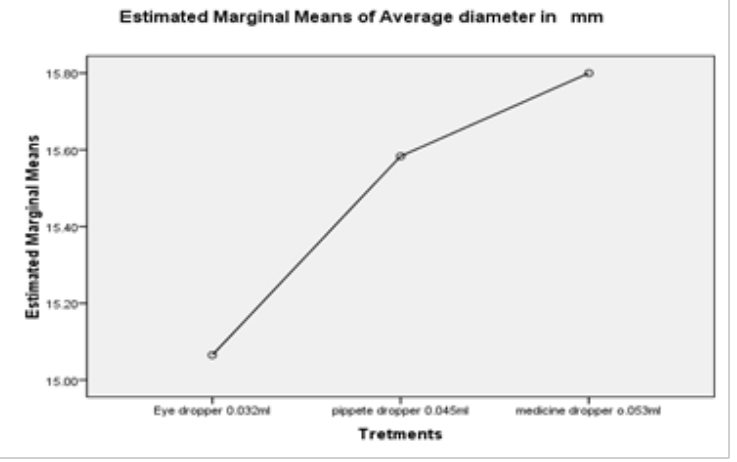

Figure 2 showing a relationship of estimated marginal means of average diameter in $\mathrm{mm}$ versus treatments.

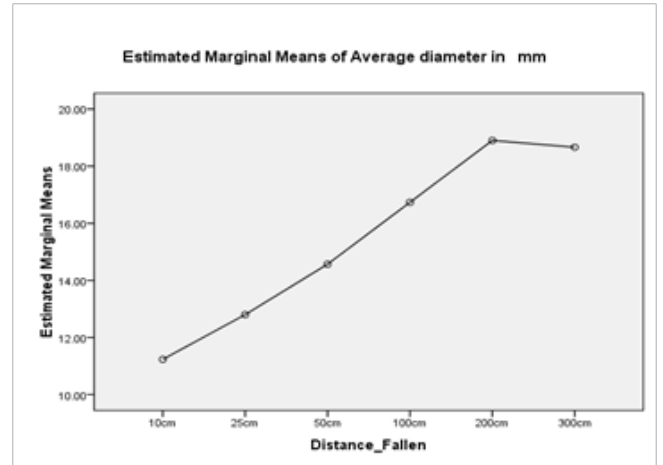

Figure 3 showing a relationship between distance fallen and estimated marginal means of average diameter. 


\section{Impact angle versus stain shape}

The afterward stage in the customary method of defining the region origin was determining the angle of impact for the bloodstains that were chose carefully. ${ }^{7}$ A blood drop struck a slightly plane horizontal surface at an angle of 90 degree resulted in an almost round drip stain while a fall at an angle less than 90 led to an extra elliptic stain. The larger the curved the angle of impact of the fallen blood drop, the more the degree of elongation of the resultant bloodstain became as the width of the bloodstain decreased and its length shortened. The similar result occurred when there was a virtual movement between the origin of a blood drop and the horizontal surface it landed on. ${ }^{4}$ Figure 4 shows that bloodstains fallen from $50 \mathrm{~cm}$ under various angels of impact. Further, Regression coefficients relationship was implied on impact angle versus stain shape formation which showed an insignificant data.

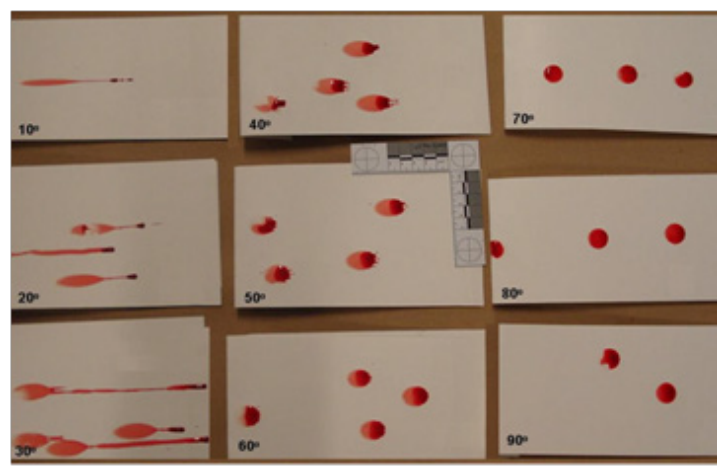

Figure 4 Regression coefficients relationship was applied on impact angle vs. stain shape formation which showed an insignificant data.

\section{Target surface effects}

The kind of surface at which blood strikes has an effect on the resulting spatter, comprising the size and look of the blood drops. To check the effect of blood stain formation at different surfaces seven surfaces i.e. flooring material, wood, card board, mild steel sheet, newspaper, flooring tiles and carpet was taken. As it was expected that there would be an insignificant relationship between the bloods stain and target surface, so were the results. Different heights were kept the same from 10,25, 50,100 and 300. Among these surfaces some of the blood stains were found round i.e. on flooring material, mild steel sheet, newspaper, cardboard and flooring tiles. But irregular stains were formed on wood and carpet (Figure 5).

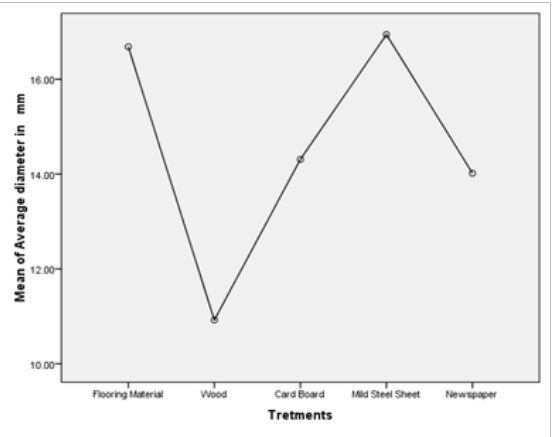

Figure $\mathbf{5}$ shows mean average diameter in $\mathrm{mm}$ versus treatments.

\section{Contact patterns}

Contact patterns on different substrates were experimented. Bloody hair and hair tip impressions were tested on the paper and ceramic tile which gave contact patterns and the direction of movement was from towards back and upward and towards the direction of movement respectively. Bloody shoe impression on paper also gave contact pattern of the left shoe and direction was found towards the direction of movement and the impression showed class characteristics. Rough bloody fabric impression on mixed polyester clothing gave an impression of contact pattern with an upward direction, having no visible class characteristics. Screwdriver impression on mixed polyester fabric and the on rough paper also showed the contact pattern having visible class characteristcs. Last but not the least Palm impression on paper of right hand also gave contact pattern forward with direction of movement.

\section{Blood in horizontal motion}

Blood drops from height of 1 meter were fallen on cardboard surface by walking upward direction. A long trail of different size of blood stains was formed. First by Walking slowly ( speed $=0.59 \mathrm{~m} / \mathrm{s}$ ) and secondly by Walking fast (speed $=0.95 \mathrm{~m} / \mathrm{s}$ ). when walked slowly more blood stains while walking with fast provided less stains.

\section{Impact spatter pattern}

Blood drops dripping into the pool of blood with height $30 \mathrm{~cm}$, volume $50 \mathrm{ml}$ showed the following results: Shape formed round stains, varying in size. Size ranged from $1.5 \mathrm{~mm}$ to $2 \mathrm{~mm}$. Max distance travelled by droplets 10.7. Stain distribution was many. Same was repeated with the change in height of $100 \mathrm{~cm}$ the resultant information was much more different except in Shape, which was round. Size ranged from less than $1 \mathrm{~mm}$ to $3.75 \mathrm{~mm}$. Max distance travelled by droplets $33 \mathrm{~cm}$ and Stain distribution was few. So it was concluded that lesser height of blood dropping showed more distribution but greater height of blood dropping showed less distribution and change in stain size as well with constant volume.

\section{Stepping into blood}

Experiment of stepping into blood with maximum and medium force was done with constant height i.e. $10 \mathrm{~cm}$ vertical target. So the obtained results were:

Stepping into blood with maximum force:

i. Spines increases with high force.

ii. Origin is at particular angle.

iii. Direction of person can be found by pattern.

iv. Spines of relatively increased in size are seen.

v. Sharp needle like streaks greater in number.

Stepping into blood with medium force:

i. Spines are less in no. at low speed.

ii. Downward flow of blood indicates that origin is at particular angle.

iii. Direction can be found by pattern. 


\section{Large volumes of falling blood}

A $2 \mathrm{ml}$ volume of blood drop was taken and was dropped from different heights of $10 \mathrm{~cm}, 25 \mathrm{~cm}, 100 \mathrm{~cm}$ and $200 \mathrm{~cm}$. Firstly, pattern from $10 \mathrm{~cm}$ fall gave regular circular large size stain of diameter $3.4 \mathrm{~cm}$. No apparent spatter shown. Radiating distance was $20 \mathrm{~cm}$ circle. A few circular secondary drops of size ranging $1 \mathrm{~mm}$ to $2 \mathrm{~mm}$ were formed. No spikes no streaks formation. Secondly, pattern from 25 $\mathrm{cm}$ fall shown irregular oblong stain of $3.6 \mathrm{~mm}$ in diameter. Radiating distance was $25 \mathrm{~cm}$ in circular area. A few circular drops of small size $1.0 \mathrm{~mm}-3.0 \mathrm{~mm}$ were formed. No spines but a few streaks were produced. Thirdly, Pattern from $100 \mathrm{~cm}$ fall gave the characteristic irregular oblong spatter of diameter $4.4 \mathrm{~cm}$.A few circular drops $2.0 \mathrm{~mm}-4.00 \mathrm{~mm}$ in close proximity with parent stain. Radiating distance of secondary stain $75 \mathrm{~cm}$. A few spines of small size were made and a few needle like streaks were also notified. Lastly, Pattern from $200 \mathrm{~cm}$ fall created irregular stain of shape, diameter $5.6 \mathrm{~cm}$ in size. Radiating distance of secondary stain is $80 \mathrm{~cm}$. Circular drops shape $2-4 \mathrm{~mm}$ in diameter. Increasing size of spines with countless streaks was produced.

\section{Discussions}

The mutual types defined for marks from dropping or proposed droplets on cloths are important, as the utmost earlier inquiries of bloodstain structure on fabric ${ }^{8}$ had concentrated on great marks with the exemption of the study by. ${ }^{6}$ For contact stains numerous ordinary standards could be recognized: Abnormality of the stain (unequal estimations). In the example of smear stains, the spreading path can be recognized by the reducing quantity of blood in the sequence of the mark and probably as of the irregular to saturate of the edges. Void planned scheme of the stain pattern everywhere stains from dropping or proposed drops incline to minimize the area withering pattern and minor drops. Moreover, those variances among contact and "dynamic" stains can be reduced with lessening dimension of the stain. As a result, micro stains of blood on a coarse texture symbolize the hardest difficulty in defining their source. A mention assembly probably is supportive and the consistency of the analysis rises with growing quantity of specific stains studied in a case. ${ }^{6}$ On planes comprising the wood and paper, the shape of the blood drop was semi-circular shape and circular shapes respectively, causing in a comparatively bulky contact angle for these faces. On flooring tile and steel sheet, the blood spread out more to form a semi-oval shape subsequently creating a smaller contact angle.

\section{Conclusion}

The consequences of the ballistic bloodstain pattern analyses gave traces about the regions of origin, the least sum of blows, the locating of the target as well as the classifications of trials. In both circumstances, important forensic decisions concerning crime reconstruction were improved by bloodstain pattern analysis based on 3D documentation and analysis and the ballistic determination of the trajectories. All the analyses regarding diameter of stain as a function of origin have shown that greater the origin of blood drop the greater will be the size. In Diameter of stain vs. distance fallen and diameter of stain vs. distance of drop as the height will also be increased the diameter of the individual stain get increase i.e. directly proportional to each other. The important and noteworthy thing is that heights, impact angles, different surfaces, forces of blood dropping and mediums, each play its different roles. Each has its worth in the determination of the crime scene.

\section{Acknowledgements}

This work was practically conducted in Punjab Forensic Science Agency, Lahore under the kind supervision of Forensic scientists. Further we are very much thankful to the administration of University of Veterinary and Animal Sciences, Lahore who's strong efforts make us possible to conduct our research in Punjab Forensic Science Agency, Lahore.

\section{Conflict of interest}

None.

\section{References}

1. Behrooz N. Bloodstain pattern analysis for determination of point of origin. Department of Mechanical and Industrial Engineering, University of Toronto. 2009.

2. Murray R. Computational and laboratory investigations of a model of blood droplet flight for forensic application. University of Ontario Institute of Technology. 2012. p. 35.

3. Nordby JJ, Abmdi D. Final analysis forensics. University Place,WA. 2009.

4. Peschel O, Kunz SN, Rothschild MA, et al. Bloodstain pattern analysis. Forensic Sci Med Pathol. 2001;7(3):257-270.

5. Shen AR, Brostow GJ, Cipolla, R. Toward automatic blood spatter analysis in crime scenes. In The Institution of Engineering and Technology Conference on Crime and Security, University of Cambridge, Department of Engineering. 2006; 378-383.

6. Karger B, Rand SP, Brinkmann B. Experimental bloodstains on fabric from contact and from droplets. Int J Legal Med. 1998;111(1):17-21.

7. Wells JK. Investigation of factors affecting the region of origin estimate in bloodstain pattern analysis. University of Canterbury. 2006.

8. White B. Bloodstain Patterns on Fabrics: The Effect of Drop Volume, Dropping Height and Impact Angle. Journal of the Canadian Society of Forensic Science. 1986;19(1):3-36. 